La locuzione seconda vita allude alla pratica della guarigione; implica un'idea di cura che sottende uno sguardo amorevole verso ciò che esiste ma impone anche, di contro, la presa d'atto che gli spazi urbani e architettonici in cui viviamo sono malati, insani, affetti da disfunzionalità che solo una ben orientata cultura del riuso può sanare. Il volume si occupa, dunque, dell'intervento sull'esistente nell'accezione più ampia possibile: intervento su un esistente qualificato; intervento su un esistente ordinario. Ciò che si intende sostenere è che le due operazioni siano in realtà due declinazioni di uno stesso tema progettuale: il lavoro su ciò che già c'è, su ciò che è materia del nostro paesaggio quotidiano. Il lavoro, in sintesi, che si qualifica per la presenza dei prefissi re- o $r$ - davanti alle sue definizioni. In questo senso lo sguardo non intende porre "paletti" disciplinari, estendendosi a considerare un'area vasta che riavvicina idealmente patrimonio alto (edilizia storica) e patrimonio basso (edilizia corrente), a favore di una cultura del recupero volta alla valorizzazione di tutte le risorse disponibili sul territorio. In questo quadro, l'obiettivo del volume è di concorrere alla definizione di un "pensiero del riuso" che superi gli steccati disciplinari e ricomponga l'insieme delle conoscenze relative ai due settori disciplinari che tradizionalmente si "contendono" la materia (progettazione e restauro), per consentire il dialogo tra i diversi operatori competenti e garantire la possibilità di un confronto nell'ambito dell'informazione sulle tecniche di intervento.

Pisana Posocco, architetto, è ricercatore presso l'Università Sapienza di Roma. Suoi scritti sono pubblicati su riviste nazionali e internazionali.. Parallelamente all'attività di ricerca svolge, con Filippo Lambertucci, una costante attività progettuale. Il lavoro dello studio è stato presentato in conferenze e pubblicato in varie riviste nazionali e internazionali.

Manuela Raitano, architetto, è ricercatore presso l'Università Sapienza di Roma. È autrice di Dentro e fuori la crisi. Percorsi di architettura italiana del secondo Novecento (Librìa, 20I2). Parallelamente all'attività di ricerca svolge una costante attività progettuale con Luca Lanini (b.e.ar.chitettura). Il lavoro dello studio è pubblicato in b.e.ar. luca lanini manuela raitano - progetti (Librìa, 2012).

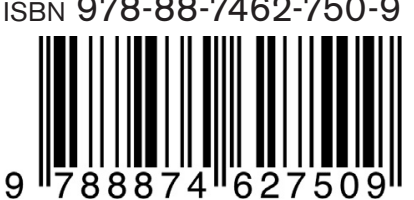

\section{La seconda vita degli edifici}

Riflessioni e progetti

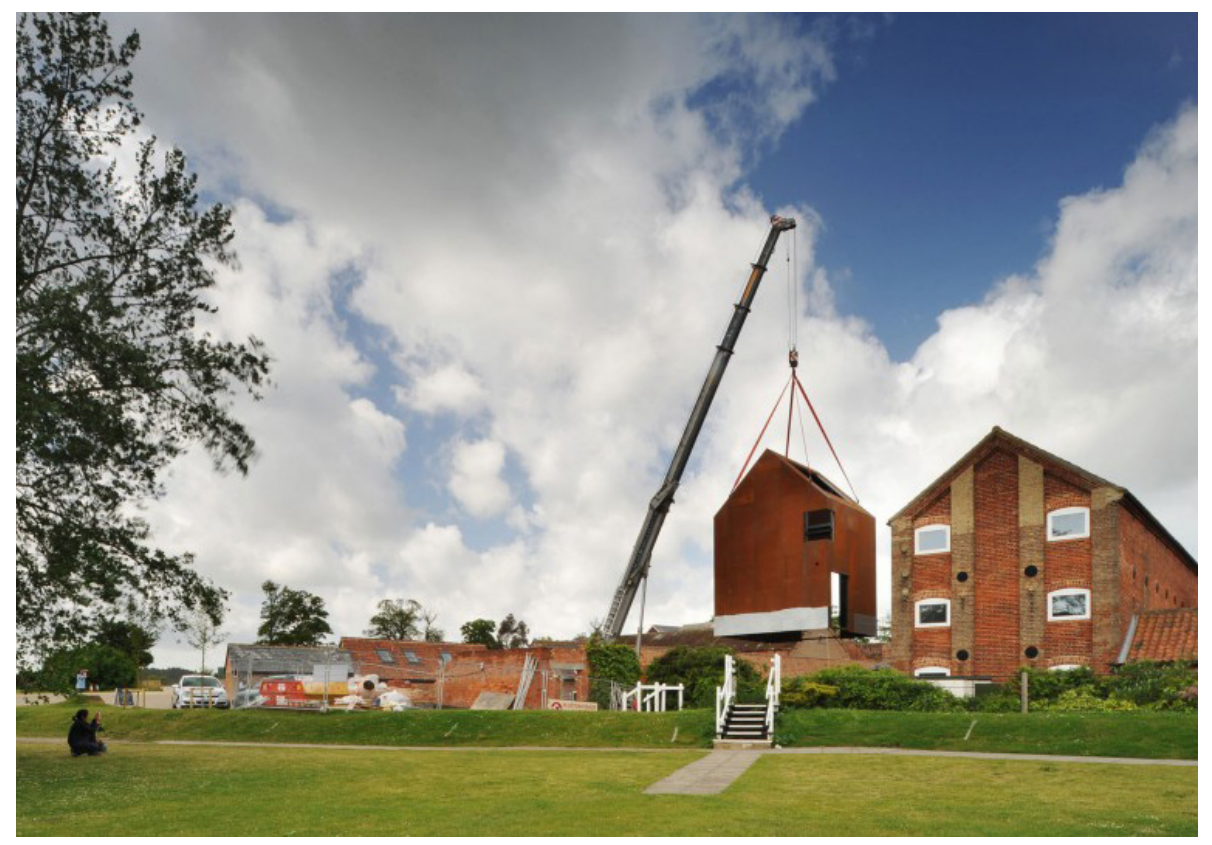

a cura di

Pisana Posocco

Manuela Raitano 


\section{La seconda vita degli edifici}

Riflessioni e progetti

a cura di

Pisana Posocco

Manuela Raitano

Quodlibet 
DiAP Dipartimento di Architettura e Progetto

Direttore Piero Ostilio Rossi

Sapienza Università di Roma

DiAP PRINT / TEORIE

Collana a cura del

Gruppo Comunicazione del DiAP

Coordinatore Orazio Carpenzano

COMITATO SCIENTIFICO

Carmen Andriani

Renato Bocchi

Alessandra Muntoni

Franco Purini

Joseph Rykwert

Andrea Sciascia

Ilaria Valente

Herman van Bergeijk

Franco Zagari

Ogni volume della collana è sottoposto alla revisione di referees esterni al Dipartimento di Architettura e Progetto scelti tra i componenti del Comitato Scientifico.
(C) 2016

Quodlibet srl

via Giuseppe e Bartolomeo Mozzi, 23

Macerata

www.quodlibet.it

PRIMA EDIZIONE

aprile 2016

ISBN

978-88-7462-750-9

IN COPERTINA

II Dovecote Studio durante la sua trasformazione, Haworth Tompkins Architects, Snape Maltings Music Campus, Suffolk

Questo libro conclude il lavoro di ricerca di Ateneo finanziamento 2012 "Rehab - progetti per l'esistente. Tecniche di guarigione dell'architettura e dei luoghi". 


\section{Indice}

7 Monumento, patrimonio, risorsa. Note introduttive alla lettura

di Pisana Posocco e Manuela Raitano

17 Progettare per l'esistente. L'architettura è guarigione Manuela Raitano

$47 \quad$ Modificare per conservare. La memoria è progetto Pisana Posocco

\section{Riflessioni}

79 Interventi sull'esistente tra tradizione e modificazione. Il caso italiano Sonia Martone

90 L'adeguamento funzionale delle strutture esistenti Maria Agostiano

$97 \quad$ Tra tempo passato e tempo presente. Il progetto di conservazione

Daniela Concas

107 Tra un muro antico e un muro nuovo. Note sul progetto della residenza negli edifici storici Amanzio Farris

115 La disciplina dell'esistente Ricardo Flores ed Eva Prats

126 La conservazione del futuro e il progetto di palinsesto. La crisi dell'invenzione della natura selvaggia Paolo Ceccon e Laura Zampieri 
138

\section{Modificare la città consolidata}

Sabrina Leone

\section{Demolizioni sensibili e rigenerazione urbana}

Alessandra Criconia

Retrofit, sostenibilità, green economy

Sergio Russo Ermolli

\section{Progetti}

Testi di D. Carfagna, M. Didomenicantonio, A. Di Giacomo, A. Felici, G. Tomassetti, L. Viola

\section{Recupero dell'ex carcere di Palencia / Spagna}

Forte di Fortezza / Bolzano, Italia

Padiglione di accesso agli scavi archeologici dell'Artemision / Siracusa, Italia

Ex mercato coperto di Ortigia / Siracusa, Italia

Copertura del parco archeologico El molinete / Cartagena, Spagna

Baroque Court Apartments / Lubiana, Slovenia

House Chargey / Chargey-les-port, Francia

Casa unifamiliare / Ragusa, Italia

Sopralzo di un complesso residenziale comunale / Cinisello Balsamo, Italia

Droogloodsen Wienenberge / Kortrijk, Belgio

Hotel Zenden / Maastricht, Paesi Bassi

Nıo-Eiras Indoor Sports Facility / Coimbra, Portogallo

Officine grandi riparazioni / Torino, Italia

Landschaftspark / Duisburg, Germania

The Great Rock / Budapest, Ungheria

Plaza de la Vila de Madrid / Barcellona, Spagna

Lent Space / New York, Stati Uniti

\section{Bibliografia ragionata}

A cura di Alessandra Di Giacomo e Giancarlo Salamone 


\title{
Demolizioni sensibili e rigenerazione urbana
}

\author{
Alessandra Criconia
}

\section{Note introduttive}

Per parlare di demolizione è bene anzitutto sgombrare il campo dai pregiudizi che il termine porta con sé. Nonostante nel corso degli ultimi anni si sia tornati a parlare di demolizione smussandone gli aspetti più radicali, non c'è verso di superare la barriera psicologica per dare alla demolizione il significato di un progetto civile che consenta di risarcire le città e il territorio dai danni di una costruzione selvaggia e fuori legge. Nell'immaginario, la demolizione continua a risuonare come qualcosa di irrimediabile, un atto finale e autoritario che al di là delle motivazioni di giusta causa, viene ancora sentito dalla collettività come una violenza inferta al bene comune. È così che paradossalmente possono trovarsi sullo stesso piano il piccone demolitore di Mussolini e le ruspe che arrivano una mattina per abbattere una casa abusiva, gli sventramenti dell'urbanistica fascista e le cariche di esplosivo utilizzate per buttare giù gli ecomostri o le talpe che bucano colline e montagne per far passare strade e ferrovie. Ma c'è demolizione e demolizione ed è riduttivo limitarla alla sola pars destruens. Come architetti e urbanisti negli ultimi tempi si sono affannati a ripetere, la demolizione ha senso solo in relazione al suo reciproco, la costruzione, e volendo usare le parole di Franco Purini, la demolizione può addirittura essere intesa come un'estensione del costruire, ovvero non un suo contrario ma "un diverso dove entra in gioco una casualità che conferisce ai suoi risultati un rilevante plusvalore emotivo e soprattutto figurativo". Un primo punto, fondamentale, è dunque messo 
in chiaro: la demolizione in architettura va considerata sempre insieme alla costruzione e mai separatamente. Questo significa che la demolizione sia quando ha un carattere distruttivo alla Walter Benjamin sia quando è sottrazione, fa sempre parte di un progetto che toglie per fare spazio a un Nuovo che non è una esatta ricostruzione di quanto c'era prima. La demolizione è dunque una questione sfaccettata che implica posizioni e scelte da fare in funzione del progetto e degli obiettivi da raggiungere.

\section{Demolizione e rigenerazione urbana. Oltre la tabula rasa}

Ora, in riferimento alla città, la demolizione ha assunto nelle sue declinazioni più recenti un valore pressoché "curativo". Adoperata in modo puntuale e mirato, essa libera gli edifici da quanto è diventato obsoleto, fatiscente, malmesso per consentire la sostituzione delle parti invecchiate e danneggiate con parti nuove, spesso riprogrammate sul piano delle destinazioni d'uso e dei materiali. Questo tipo di "demolizione finalizzata" è uno dei momenti salienti della rigenerazione e di un progetto di trasformazione urbana che interviene sull'esistente nel rispetto dell'identità dei luoghi e di chi vi abita.

Su questo fronte anche una scuola notoriamente legata alla tabula rasa com'è quella francese e che negli anni '70 è stata protagonista di un'operazione di demolizione radicale nel centro di Parigi, l'abbattimento degli storici mercati delle Halles per fare spazio al Beaubourg e realizzare il nodo di scambio di Châtelet, sostiene ora l'urbanesimo sensibile, azioni mirate di recupero e riciclo dell'esistente per favorire una ri-architetturizzazione tanto fisica quanto sociale dei contesti urbani e ambientali. Il punto di fondo è che le demolizioni anche quando sono state adoperate in chiave civile hanno intaccato o leso, talvolta in modo irreversibile, il legame di appartenenza degli abitanti al loro luogo di vita, con risvolti negativi sul fronte della cura della città. In un suo saggio, la storica delle forme urbane Françoise Choay attribuisce la rimozione dei 
vincoli spaziali, temporali e corporei e la conseguente perdita di memoria antropologica alla dissociazione della coppia demolizione/conservazione. "La demolizione che oggi si pretende di giustificare nel nome della necessità storica non è più la demolizione tradizionale - l'inverso di un costruire. È una negatività senza positivo. La tabula rasa, una volta rioccupata, rimane irrimediabilmente tabula rasa. Gli sviluppi egemoni delle infrastrutture a rete e degli interventi a scala territoriale tendono a soppiantare e a condannare quella scala costruttiva di prossimità e di urbanità che contribuiva a fondare la nostra identità e la nostra legittimità antropologica. Ed è proprio questa scala, che una falsa coscienza storica ed un falso pudore non dovrebbero impedirci di chiamare "umana", che stiamo dimenticando e disimparando ogni giorno di più".

Alle demolizioni troppo drastiche, vanno preferite le piccole demolizioni fatte in relazione al contesto e alla sua identità. A tale proposito è interessante notare come anche un architetto illuminista di fede moderna qual è Jean Nouvel, abbia impostato il suo progetto Grand Paris sul principio del genius loci "...è urgente risvegliare e invitare un vecchio genio che abbiamo stupidamente congedato nella prima metà del xx secolo: il genio del luogo. È un poeta, un filosofo. Parla di piaceri e di felicità. Ci ricorda che per salvare Parigi dalla volgarità e dalla profanazione, bisogna farsi incantare dalla luce dell'Ile de France, guardare i cieli, le nuvole, le foreste, i campi..., vivere le rive e il fiume, i ponti di Parigi, le quais, le péniches..., alzare gli occhi anche sulla grigia distesa dei tetti di zinco, cielo al contrario da cui emergono le silhouette degli edifici singolari, simboli del passato. Ma questo genio si interesserà anche a tutto ciò che è arrivato in sua assenza...".

Il progetto di Jean Nouvel e del suo gruppo di cui fanno parte anche l'urbanista Michel Cantal-Dupart e l'architettoingegnere Jean-Marie Duthilleul ambedue dell'agenzia AREP, è un insieme di strategie puntuali che non hanno come fine l'abbattimento delle banlieue ma la loro riconfigurazione attraverso interventi mirati sui volumi delle stecche, le cosiddette barre, nell'ottica di un restauro del moderno in chiave contem- 
poranea. Le demolizioni sono in questo caso sempre associate a delle operazioni di vera e propria ristrutturazione dell'edificio e di aumento delle cubature mediante svuotamento del basamento, inserimento di logge e terrazze nello spessore della facciata, rimodellazione delle coperture piane, creazione di piani mansardati. La nuova demolizione, in ragione inversa a quanto ipotizzato dalla Modernità, non mira più a fare tabula rasa e a cancellare le tracce di ciò che è stato per fondare il Nuovo, ma intende essere parte di un processo di trasformazione di più ampio respiro che prende inizio dalla realtà dell'esistente per creare altre specie di spazi, ibridando il vecchio con il nuovo "secondo una logica di gioco, di reiterazione e di ricombinamento". Si tratta di intendere la demolizione in una chiave de-costruttiva di smontaggio e rimontaggio degli elementi dell'architettura con esiti formali ben diversi dalle schegge di Zaha Hadid e Daniel Libeskind o dagli inviluppi biomorfi di Frank O. Gehry e di Greg Lynn. Oggi la demolizione è una strategia della sostenibilità finalizzata al recupero degli edifici e della periferia che opera delle rimozioni per creare dispositivi di risparmio energetico e migliorare la qualità dell'abitare senza cedere alle mistificazioni dell'estetica.

\section{Uno sguardo retrospettivo}

In fondo una visione sostenibile e pragmatica della demolizione era emersa fin dagli anni '8o. La conversione di vecchi distretti industriali divenuti obsoleti o di intere parti di città compromesse in nuovi quartieri per adattare la città alle esigenze della società globale aveva già in sé l'idea di una riqualificazione sostenibile senza tabula rasa, anche se alcuni dei casi più emblematici come la Vila Olimpica a Barcellona e la grande operazione di Euralille sono stati realizzati sacrificando pezzi importanti di città. Ma ci sono stati altri casi come la bonifica del bacino industriale della Ruhr e la sua riconversione in parco o gli interventi di sustainable re-furbishing nella city di Londra che hanno usato la demolizione in maniera selettiva e 
“gentile". Anche in Italia pur con il solito ritardo ci si è posti la questione di una demolizione in chiave sostenibile, purtroppo il cambiamento politico e culturale non c'è stato, nonostante alcune importanti iniziative datate anni ' 90 tra cui un disegno di legge per la rottamazione del patrimonio fatiscente presentato dal primo governo Prodi e il convegno "Il progetto della sottrazione" promosso da Paola Falini e Antonino Terranova con il sostegno dell'ANCsA, l'Associazione Nazionale dei Centri Storici e Artistici, che hanno chiamato la comunità degli urbanisti e degli architetti a interrogarsi sul significato teorico e pratico della demolizione. Per anni alcune delle questioni sollevate dai promotori del convegno - è possibile pensare la demolizione come tecnica progettuale per risanare e risarcire contesti ambientali altamente compromessi? la demolizione può essere intesa come strategia di risignificazione simbolica e riconfigurazione formale della città contemporanea? quali sono i vantaggi economici e ambientali di una demolizione ragionata nei piani di recupero del patrimonio edilizio? - hanno continuato a risuonare nel dibattito nazionale. Così come il tentativo di legge di un piano di "rottamazione edilizia" ha favorito la diffusione degli studi di rigenerazione architettonica e urbana delle periferie specialmente di quelle delle città del Mezzogiorno d'Italia.

\section{Poetiche della demolizione}

Nello scenario attuale condizionato dalla fragilità dell'economia e da valori demografici squilibrati, la demolizione è tornata alla ribalta come strategia di recupero e di salvaguardia ambientale che però è attenta alle identità dei luoghi e alle appartenenze. È questa una demolizione multifocale che fa riferimento a una estetica di "postproduzione" secondo le tesi di Nicolas Bourriaud, che mira a una riprogrammazione delle forme e degli spazi esistenti a partire dalla lettura critica del "testo" urbano e dal fatto che la demolizione è già dentro il paesaggio della città, nel deperimento dei suoi edifici e dei suoi 
luoghi. D'altro canto come negare il degrado delle periferie di Milano, di Roma, di Napoli, di Palermo, la triste monotonia delle banlieue di Parigi, la decadenza di molti edifici di Manhattan, la miseria delle favelas di San Paolo, di Rio de Janeiro, di Salvador e di molte città sudamericane, africane, asiatiche? Nulla appare più lontano e consumato del mito moderno della "città bella". Analogamente agli organismi biologici, la città è soggetta all'azione del tempo e degli agenti che operano invisibili, erodendo e spezzando i legami, facendo dissolvere nell'aria tutto ciò che è solido per usare la celebre frase di Karl Marx. Ma al di là dei ragionamenti che si possono avviare a partire da queste considerazioni, è importante sottolineare lo shock "estetico" prodotto dall'immagine disgregata della città e che sta modificando l'idea del bello e del brutto. È per via di questo cambiamento che è possibile attribuire un valore di bella opera all'intervento di ristrutturazione del Palais de Tokyo fatto dagli architetti Lacaton \& Vassal che hanno usato la demolizione non soltanto come tecnica del progetto ma anche come linguaggio. Lacaton \& Vassal hanno infatti scelto di lasciare a nudo la struttura muraria e tecnologica senza nascondere i segni del tempo e della mancanza di manutenzione per mettere in mostra, così come si fa con le opere d'arte, l'edificio stesso, attribuendo così valore estetico alle pareti crepate con i segni delle demolizioni, le tracce degli impianti elettrici, le tubature del condizionamento. È evidente che la nuova destinazione d'uso del Palais de Tokyo in un sito della creazione contemporanea è stata complice della scelta poetica degli architetti: la conversione di un edificio monumentale e senza particolari qualità qual è il Palais de Tokyo in una sede espositiva di arte contemporanea della postproduzione ha giocato di sponda con l'uso estetico della demolizione. Sulla scia del Palais de Tokyo, si è diffusa la moda di adottare il linguaggio della demolizione in luogo della demolizione totale. È così che anche un ecomostro come la base sottomarina di Saint Nazaire - un bunker di $480.000 \mathrm{mc}$ di cemento pluriarmato, lungo 290 $\mathrm{m}$ per $\mathrm{I} 50$ e alto $\mathrm{I} 6 \mathrm{~m}$ con una copertura di $8 \mathrm{~m}$ di spessore è stato recuperato con un intervento leggero, quasi invisibile, 
che lascia inalterata la forma massiva. Consapevole dell'impossibilità di demolire un tale volume di cemento armato, lo studio di progettazione LIN vincitore del concorso ha preferito creare delle relazioni tra interno ed esterno con un intervento "chirurgico" che lascia inalterato il carattere specifico del bunker. I risultati sono però particolarmente suggestivi e inducono a pensare che il linguaggio della demolizione sia la strada giusta per ricostruire la coppia demolizione/conservazione e ricostruire quella memoria antropologica che la Choay ritiene indispensabile per fondare la nostra identità di cittadini. 